EUROPEAN JOURNAL OF PURE AND APPLIED MATHEMATICS

Vol. 13, No. 1, 2020, 33-47

ISSN 1307-5543 - www.ejpam.com

Published by New York Business Global

\title{
Higher order nonlocal boundary value problems at resonance on the half-line
}

\author{
S.A. Iyase ${ }^{1}$, A.A. Opanuga ${ }^{2, *}$ \\ ${ }^{1}$ Department of Mathematics, College of Science and Technology, Covenant University, \\ Ota, Ogun State, Nigeria
}

Abstract. This paper investigates the solvability of a class of higher order nonlocal boundary value problems of the form

$$
u^{(n)}(t)=g\left(t, u(t), u^{\prime}(t) \cdots u^{(n-1)}(t)\right), \text { a.e. } t \in(0, \infty)
$$

subject to the boundary conditions

$$
\begin{aligned}
u^{(n-1)}(0) & =\frac{(n-1) !}{\xi^{n-1}} u(\xi), u^{(i)}(0)=0, i=1,2, \ldots, n-2, \\
u^{(n-1)}(\infty) & =\int_{0}^{\xi} u^{(n-1)}(s) d A(s)
\end{aligned}
$$

where $\xi>0, g:[0, \infty) \times \Re^{n} \longrightarrow \Re$ is a Caratheodory's function,

$A:[0, \xi] \longrightarrow[0,1)$ is a non-decreasing function with $A(0)=0, A(\xi)=1$. The differential operator is a Fredholm map of index zero and non-invertible. We shall employ coicidence degree arguments and construct suitable operators to establish existence of solutions for the above higher order nonlocal boundary value problems at resonance.

2020 Mathematics Subject Classifications: 34B40, 34B15

Key Words and Phrases: Higher order, Resonance, Coincidence degree, Nonlocal boundary value problem, Half-Line

\section{Introduction}

In this paper, we study the existence of solutions for the higher order boundary value problems.

$$
u^{(n)}(t)=g\left(t, u, u^{\prime}(t) \ldots u^{n-1}(t)\right) \text { a.e. } t \in(0, \infty)
$$

* Corresponding author.

DOI: https://doi.org/10.29020/nybg.ejpam.v13i1.3539

Email addresses: samuel.iyase@covenantuniversity.edu.ng (S.A. Iyase), abiodun.opanuga@covenantuniversity.edu.ng (A.A. Opanuga) 
subject to the boundary conditions

$$
\begin{array}{r}
u^{(n-1)}(0)=\frac{(n-1) !}{\xi^{n-1}} u(\xi), u^{(i)}(0)=0, i=1,2, \ldots, n-2, \\
u^{(n-1)}(\infty)=\int_{0}^{\xi} u^{(n-1)}(s) d A(s)
\end{array}
$$

where $\xi>0, g:[0, \infty) \times \Re^{n} \longrightarrow \Re$ is a Caratheodory's function and $A:[0, \xi] \longrightarrow[0,1)$ is a non-decreasing function with $A(0)=0, A(\xi)=1$. The integral is the Riemann-Stieltjes integral. Boundary value problems such as (1)-(2) with nontrivial Kernels are called resonance problems. To the best of our knowledge higher order boundary value problems on infinite intervals at resonance have not received much attention in the Literature especially those with integral boundary conditions. Most papers focused on boundary value problems at resonance on finite intervals especially for second and third order boundary value problems. For some results in this direction see $[4,5,6,7,8,9,10$, $11,12,13,14,15,17,18,19]$ and references therein. Nonlocal boundary value problems were first studied in [3] by Bicadze and Samarskii. In a recent paper [10] Karakostas and Tsamatos considered the following nonlocal boundary value problem.

$$
\begin{gathered}
x^{\prime \prime}(t)+q(t) f\left(x(t), x^{\prime}(t)\right)=0, t \in(0,1) \\
x(0)=0, x^{\prime}(1)=\int_{0}^{1} x^{\prime}(s) d g(s)
\end{gathered}
$$

under the nonresonance condition $0=g(0) \leq g(1)<1$. They used Krasnoselskii's fixed point theorem in establishing existence of solutions. In [13] Lin derived existence results for the nonlocal boundary value problem.

$$
\begin{gathered}
x^{\prime \prime}(t)=f\left(t, x(t), x^{\prime}(t)\right), t \in(0,1) \\
x(0)=\alpha(\xi), x^{\prime}(1)=\int_{0}^{1} x^{\prime}(s) d g(s)
\end{gathered}
$$

under the resonant condition $g(1)=1$

The main purpose of this paper is to provide new sufficient conditions that guarantees existence of solutions to (1)-(2). Our investigation will be based on the coincidence degree theory of Mawhin [17].

The main motivation for this article is the recent paper of Frioui, Guezane-Lakoud and Khaldi [7]. The authors obtained existence results for the problem

$$
\begin{gathered}
x^{(n)}(t)=f(t, x(t)), t \in(0, \infty) \\
x^{(i)}(0)=0, i:=0,1 \ldots n-2, x^{(n-1)}(\infty)=\frac{n !}{\xi^{n}} \int_{0}^{\xi} x(t) d t
\end{gathered}
$$

where $f:[0, \infty) \times \Re \longrightarrow \Re$ is a given function satisfying certain conditions. There is so far little research with regards to (1)-(2), therefore it is important to investigate it. We 
also note that (1)-(2) is more general than (3)-(4).

In section 2, we provide some background definitions, Lemmas and the coincidence degree theorem of Mawhin [17], section 3 will be devoted to proving the main existence results.

\section{Preliminaries}

In this section, we recall some background definitions and the coincidence degree theorem [17]. We shall also provide compactness criterion for continuous vector-valued functions on unbounded domains. First, we give some background results from coincidence degree theory.

\section{Definition 2.1:}

Definition 1. Let $X$ and $Z$ be real Banach Spaces. A linear mapping $L: \operatorname{dom} L \subset X \longrightarrow$ $Z$ is said to be a Fredholm mapping if

(i) $\operatorname{Ker} L$ has finite dimension.

(ii) $\operatorname{ImL}$ is closed and has a finite codimension.

In this case, the Fredholm index is the integer

$$
\operatorname{Ind} L=\operatorname{dim} k e r L-\operatorname{codim} \operatorname{Im} L .
$$

In this work, we shall utilise Fredholm mappings of index zero. If $L$ is a Fredholm mapping of index zero, then there exist continuous projections

$P: X \longrightarrow X$ and $Q: Z \longrightarrow \longrightarrow Z$

such that

$\operatorname{Im} P=\operatorname{ker} L, \operatorname{ker} Q=\operatorname{Im} L$ and $X=\operatorname{ker} L \oplus \operatorname{ker} P$

$Z=\operatorname{Im} L \oplus \operatorname{Im} Q$ and the mapping

$\left.L\right|_{\operatorname{dom} L \cap \operatorname{ker} P}: \operatorname{dom} L \cap \operatorname{ker} P \longrightarrow \operatorname{ImL}$ is invertible.

We denote the inverse of $\left.L\right|_{\text {dom } L \cap \operatorname{ker} P}$ by $K_{p}: \operatorname{Im} L \longrightarrow \operatorname{dom} L \cap \operatorname{ker} P$. We designate the generalised inverse of $L$ given by $K_{P, Q}: Z \longrightarrow d o m L \cap \operatorname{ker} P$ as $K_{P, Q}=K_{P}(I-Q)$.

Definition 2.2: The map $g:[0, \infty) \times \Re^{n} \longrightarrow \Re$ is $L^{1}[0, \infty)$-Caratheodory, if the following conditions are satisfied.

(i) For each $u \in \Re^{n}, f(t, u)$ is Lebesgue measurable

(ii) For a.e. $t \in[0, \infty)$, there exists $\varphi_{r} \in L^{1}[0, \infty)$ such that for a.e. $t \in[0, \infty)$ and every $u$ such that $|u| \leq r$ we have $|f(t, u)| \leq \varphi_{r}(t)$.

Let $X=\left\{u \in C^{n-1}[0, \infty), \lim _{t \rightarrow \infty} e^{-t}\left|u^{(i)}(t)\right|\right.$ exists, $\left.0 \leq i \leq n-1, u^{(n)}(t) \in L^{1}[0, \infty)\right\}$ endowed with the norm

$$
\|x\|=\max _{0 \leq i \leq n-1}\left(\sup _{t \in[0, \infty)} e^{-t}\left|u^{(i)}(t)\right|\right)
$$


Then $X$ is a Banach space.

Theorem 2.1 [2] a Let $\mathrm{F}$ be a subset of $C_{\infty}=\left\{y \in C([0, \infty)), \lim _{t \rightarrow \infty)} y(t)\right.$ exists that is equipped with the norm $\|y\|_{\infty}=\sup _{t \in[0, \infty)}|y(t)|$

Then $\mathrm{F}$ is relatively compact if the following conditions hold.

(i) F is bounded in $X$

(ii) The functions belonging to $\mathrm{F}$ are equicontinuous on any compact subinterval of $[0, \infty)$

(iii) The functions from $\mathrm{F}$ are equiconvergent at infinity.

The following adaptation of the above theorem will be used to establish the compactness of $K_{p}(I-Q)$

\section{Lemma 2.1 [7]:}

Let $D \subset X$, then $D$ is relatively compact in $X$ if the following conditions hold.

(i) $D$ is bounded in $X$

(ii) The family $W^{i}=\left\{\psi_{i}: \psi_{i}(t)=e^{-t} u^{(i)}(t), t \geq 0, u \in D\right\}$ is equicontinuous on any compact subinterval of $[0, \infty)$ for $i=0, \ldots, n-1$.

(iii) The family $W^{i}=\left\{\psi_{i}: \psi_{i}(t)=e^{-t} u^{(i)}(t), t \geq 0, u \in D\right\}$ is equiconvergent at infinity for $i=0,1, \ldots, n-1$.

Let $Z=L^{1}[0, \infty)$ with the norm $\|y\|_{1}=\int_{0}^{\infty}|y(t)| d t$ for $y \in Z$. We denote $A C_{l o c}[0, \infty)$ as the space of locally absolutely continuous functions on $[0, \infty)$. We define $L$ to be the linear operator from $\operatorname{dom} L \subset X \rightarrow Z$ with

$$
\begin{aligned}
\operatorname{dom} L & =\left\{u \in X: u^{(n-1)}(t) \in A C_{l o c}[0, \infty), u^{(i)}(0)=0, i=1,2, \ldots, n-2\right. \\
u^{(n-1)}(0) & \left.=\frac{(n-1) !}{\xi^{n-1}} u(\xi), u^{(n-1)}(\infty)=\int_{0}^{\xi} u^{(n-1)}(s) d A(s), u^{(n)} \in Z\right\}
\end{aligned}
$$

and $L u(t)=u^{(n)}(t), u \in d o m L, t \in[0, \infty)$. We define $N: X \longrightarrow Z$ by setting

$$
N u(t)=g\left(t, u(t), u^{\prime}(t) \cdots u^{(n-1)}(t)\right), t \in[0, \infty)
$$

We can then write (1)-(2) as

$$
L u=N u
$$

\section{Definition 2.3:}

Definition 2. Let $L: \operatorname{dom} L \subset X \longrightarrow Z$ be a Fredholm mapping, $E$ a metric space and $N: E \longrightarrow Z$ be a mapping: $N$ is said to be $L$-compact on $E$ if $Q N: E \longrightarrow Z$ and $K_{P, Q} N: E \longrightarrow Z$ are compact on $E . N$ is called completely continuous if it is L-compact on every bounded $E \subset X$. 

[17].

The existence of solution to (5) will be guaranteed by the following theorem of Mawhin

Theorem 2.2 [16]: Let $\Omega \subset X$ be , and $L$ be a Fredholm mapping of index zero and $N$ be $L$-compact on $\bar{\Omega}$. Assume that the following conditions are satisfied.

(1) $L u \neq N u$ for every $(u, \lambda) \in[(\operatorname{dom} L \backslash \operatorname{ker} L) \cap \partial \Omega] \times(0,1)$

(2) $N u \notin \operatorname{ImL}$ for every $u \in \operatorname{ker} L \cap \partial \Omega$

(3) $\operatorname{deg}\left(\left.Q N\right|_{\partial \Omega \cap \operatorname{ker} L}, \Omega \cap \operatorname{ker} L, 0\right) \neq 0$ where $Q: Z \longrightarrow Z$ is a projection such that $\operatorname{Im} L=\operatorname{ker} Q$

Then the equation $L u=N u$ has at least one solution in $\operatorname{dom} L \cap \bar{\Omega}$.

Lemma 2.2: If $u^{(n-1)}(0)=\frac{(n-1) !}{\xi^{n-1}} u(\xi), A(\xi)=1, A(0)=0$,

$u^{(n-1)}(\infty)=\int_{0}^{\xi} u^{(n-1)}(s) d A(s), u^{(i)}(0)=0, i=1,2, \ldots, n-2$ then

(i) $\operatorname{ker} L=\left\{u \in \operatorname{dom} L: u=d t^{n-1}, d \in \Re, t \in(0, \infty)\right\}$

(ii) $\operatorname{ImL}=\left\{y \in Z: \int_{0}^{\infty} y(\tau) d \tau-\int_{0}^{\xi} \int_{0}^{s} y(v) d v d A(s)=0\right\}$.

\section{Proof:}

(i) Let $u \in \operatorname{ker} L$, then $u^{(n)}(t)=0$ for a.e. $t \in[0, \infty)$. Since $u^{(i)}(0)=0$ for $i=$ $1.2 \ldots, n-2$ and using (5) we derive that $u(t)=d t^{n-1}$. Thus

$$
\operatorname{ker} L=\left\{u \in X: u(t)=d t^{n-1}\right\}
$$

(ii) We next show that

$$
\operatorname{ImL}=\left\{y \in Z: \int_{0}^{\infty} y(s) d s-\int_{0}^{\xi} \int_{0}^{s} y(\tau) d \tau d A(s)=0\right\} .
$$

We consider the problem

$$
u^{(n)}(t)=y(t), \quad y \in Z
$$

We prove that (6) has a solution $u(t)$ satisfying

$$
u^{(n-1)}(0)=\frac{(n-1) !}{\xi^{n-1}} u(\xi), u^{(i)}(0)=0, \quad i=1,2, \ldots, n-2, u^{n-1}(\infty)=\int_{0}^{\xi} u^{(n-1)}(s) d A(s)
$$

If and only if

$$
\int_{0}^{\infty} y(\tau) d \tau-\int_{0}^{\xi} \int_{0}^{s} y(v) d v d A(s)=0
$$


Suppose (6) has a solution $u(t)$ satisfying (5). We show that this solution satisfies (7).

From (5) we obtain

$$
\begin{aligned}
u(t) & =u(0)+\frac{u^{(n-1)}}{(n-1) !} t^{n-1}+\int_{0}^{t} \int_{0}^{\tau_{n}} \ldots \int_{0}^{\tau_{2}} y(\tau) d \tau_{1}, \ldots, d \tau_{n} \\
u^{(n-1)}(t) & =u^{(n-1)}(0)+\int_{0}^{t} y(\tau) d \tau \\
u^{(n-1)}(\infty) & =u^{(n-1)}(0)+\int_{0}^{\infty} y(\tau) d \tau=\int_{0}^{\xi}\left[u^{(n-1)}(0)+\int_{0}^{s} y(\tau) d \tau\right] d A(s) \\
& =u^{(n-1)}(0) A(\xi)+\int_{0}^{\xi} \int_{0}^{s} y(\tau) d \tau d A(s) \\
& =u^{(n-1)}(0)+\int_{0}^{\xi} \int_{0}^{s} y(\tau) d \tau d A(s)
\end{aligned}
$$

and hence

$$
\int_{0}^{\infty} y(\tau) d \tau-\int_{0}^{\xi} \int_{0}^{s} y(v) d v d A(s)=0 .
$$

If however, (7) holds then setting

$$
u(t)=\frac{u^{(n-1)}(0)}{(n-1) !} t^{n-1}+\int_{0}^{t} \int_{0}^{\tau_{n}} \cdots \int_{0}^{\tau_{2}} y\left(\tau_{1}\right) d \tau_{1}, \cdots, d \tau_{n}
$$

We conclude that $u(t)$ is a solution of (6) satisfying (7).

Lemma 2.3 The mapping $L: \operatorname{dom} L \subset X \longrightarrow Z$ is a Fredholm mapping of index zero and furthermore the linear continuous projector $Q: Z \longrightarrow Z$ can be defined as

$$
Q y=h(t)\left[\int_{0}^{\infty} y(s) d s-\int_{0}^{\xi} \int_{0}^{s} y(\tau) d \tau d A(s)\right]
$$

where

$$
h(t)=\frac{e^{-t}}{\int_{0}^{\xi} e^{-s} d A(s)}
$$

The linear operator $K_{p}: \operatorname{Im} L \rightarrow \operatorname{dom} L \cap \operatorname{ker} P$, the inverse of $L \mid d o m L \cap k e r p$ can be defined as

$$
K_{p} y=\int_{0}^{t} \int_{0}^{\tau_{n}} \cdots \int_{0}^{\tau_{2}} y\left(\tau_{1}\right), d \tau_{1} \ldots d \tau_{n}-\int_{0}^{\xi} \int_{0}^{\tau_{n}} \cdots \int_{0}^{\tau_{2}} y\left(\tau_{1}\right) d \tau_{1} \cdots d \tau_{n}
$$

with

$$
\left\|K_{p} y\right\| \leq D_{n}\|y\|_{1}
$$


where

$$
D_{n}=\max \left[2 \sup _{t \in[0, \infty)} e^{-t} t^{n-1}, \max _{1 \in i \leq n-1}\left(\sup _{t \in[0, \infty)} e^{-t} t^{n-1-i}\right)\right]
$$

Proof: For $y \in Z$, we define the projection $Q y$ as:

$$
Q y=h(t)\left[\int_{0}^{\infty} y(v) d v-\int_{0}^{\xi} \int_{0}^{s} y(\tau) d \tau d A(s)\right]
$$

where

$$
h(t)=\frac{e^{-t}}{\int_{0}^{\xi} e^{-s} d A(s)} \neq 0
$$

Then we have

$$
\begin{aligned}
Q^{2} y=Q(Q y) & =h(t)\left[\int_{0}^{\infty} y(v) d v-\int_{0}^{\xi} \int_{0}^{s} y(\tau) d \tau d A(s)\right] \cdot \frac{\int_{0}^{\xi} e^{-s} d A(s)}{\int_{0}^{\xi} e^{-s} d A(s)} \\
& =Q y
\end{aligned}
$$

This implies that $Q$ is a projection.

Let $y_{1}=y-Q y$ i.e. $y_{1} \in \operatorname{ker} Q$ then

$$
\begin{aligned}
& \int_{0}^{\infty} y_{1}(v) d v-\int_{0}^{\xi} \int_{0}^{s} y_{1}(\tau) d \tau d A(s) \\
= & {\left[\int_{0}^{\infty} y(v) d v-\int_{0}^{\xi} \int_{0}^{s} y(\tau) d \tau d A(s)\right]\left[1-\frac{\int_{0}^{\xi} e^{-s} d A(s)}{\int_{0}^{\xi} e^{-s} d A(s)}\right]=0 }
\end{aligned}
$$

Therefore, $y_{1} \in \operatorname{Im} L$ and hence $Z=\operatorname{Im} L+\operatorname{Im} Q$.

Since $\operatorname{Im} L \cap \operatorname{Im} Q=\{0\}$ we obtain $Z=\operatorname{Im} Q \oplus \operatorname{Im} L$.

This implies that $\operatorname{dim} \operatorname{ker} L=\operatorname{dim} \operatorname{Im} Q=1$.

Hence, $L$ is a Fredholm operator of index zero.

Let $P: X \longrightarrow X$ be defined by

$$
P u=\frac{u^{(n-1)}(0) t^{n-1}}{(n-1) !}
$$

We define

$$
\begin{gathered}
K_{p}: \operatorname{Im} L \longrightarrow d o m L \cap \operatorname{ker} P \text { as } \\
K_{p} y=\int_{0}^{t} \int_{0}^{\tau_{n}} \cdots \int_{0}^{\tau_{n}} y\left(\tau_{1}\right) d \tau_{1} \cdots d \tau_{n}-\int_{0}^{\xi} \int_{0}^{\tau_{n}} \cdots \int_{0}^{\tau_{2}} y\left(\tau_{1}\right) d \tau_{1} \cdots d \tau_{n}
\end{gathered}
$$


For $y \in \operatorname{ImL},\left(L K_{p}\right) y(t)=\left[\left(K_{p} y\right)(t)\right]^{n}=y(t)$ and for $u \in \operatorname{dom} L \cap \operatorname{ker} P$ and noting that $u^{(i)}(0)=0, i=1.2, \ldots, n-2$

$$
\left(K_{p} L\right) u(t)=u(t)-\frac{u^{(n-1)}(0) t^{n-1}}{(n-1) !}-u(\xi)+\frac{u^{(n-1)}(0) \xi^{n-1}}{(n-1) !}
$$

Since $u \in d o m L \cap \operatorname{ker} P, \quad P u=\frac{u^{(n-1)}(0)}{(n-1) !} t^{n-1}=0$.

Also since $u^{(n-1)}(0)=\frac{(n-1) !}{\xi^{n-1}} u(\xi)$ we derive

$\left(K_{p} L\right) u(t)=u(t)$

Thus $K_{p}=\left(\left.L\right|_{\operatorname{dom} L \cap \operatorname{ker} P}\right)^{-1}$

$$
\begin{aligned}
e^{-t}\left|\left(K_{p} y\right)(t)\right| & =e^{-t}\left|\left[\int_{0}^{t} \int_{0}^{\tau_{n}} \cdots \int_{0}^{\tau_{n}} y\left(\tau_{1}\right) d \tau_{1} \cdots d \tau_{n}-\int_{0}^{\xi} \int_{0}^{\tau_{n}} \cdots \int_{0}^{\tau_{2}} y(\tau) d \tau_{1} \cdots d \tau_{n}\right]\right| \\
& \leq e^{-t}\left[\int_{0}^{t} \int_{0}^{\tau_{n}} \cdots \int_{0}^{\tau_{2}}\left|y\left(\tau_{1}\right)\right| d \tau_{1} \cdots d \tau_{n}+\int_{0}^{\xi} \int_{0}^{\tau_{n}} \cdots \int_{0}^{\tau_{2}}\left|y\left(\tau_{1}\right)\right| d \tau_{1} \cdots d \tau_{n}\right] \\
& \leq \sup _{t \in[0, \infty)} e^{-t} t^{n-1} \int_{0}^{\infty}|y(s)| d s+\sup _{t \in[0, \infty)} e^{-t} t^{n-1} \int_{0}^{\infty}|y(s)| d s \\
& \leq 2 \sup _{t \in[0, \infty)} e^{-t} t^{n-1}\|y\|_{1}
\end{aligned}
$$

For $1 \leq i \leq n-1$ we have

$$
\begin{aligned}
e^{-t}\left|\left(K_{p} y\right)^{(i)}(t)\right| & =\frac{e^{-t}}{(n-1-i) !}\left|\int_{0}^{t}(t-s)^{n-1-i} y(s) d s\right| \\
& \leq e^{-t} t^{n-1-i} \int_{0}^{\infty}|y(s)| d s
\end{aligned}
$$

We therefore conclude that

$$
\begin{aligned}
\left\|K_{p} y\right\| & \leq \max \left[2 \sup _{t \in[0, \infty)} e^{-t} t^{n-1}, \max _{1 \leq i \leq n-1}\left(\sup _{t \in[0, \infty)} e^{-t} t^{n-1-i}\right)\right]\|y\|_{1} \\
& =D_{n}\|y\|_{1}
\end{aligned}
$$

where $D_{n}=\max \left[2 \sup _{t \in[0, \infty)} e^{-t} t^{n-1}, \max _{1 \leq i \leq n-1}\left(\sup _{t \in[0, \infty)} e^{-t} t^{n-1-i}\right)\right]$

Lemma 2.4 If $g$ is a Caratheodory's function and $E \subset X$ is a bounded open subset of $X$, such that $\operatorname{dom} L \cap \bar{E} \neq \phi$ then $N$ is $L$-compact, where $E$ denotes the closure of $E$

Proof: Let $E \subset X$ with $r=\sup \{\|u\|: u \in \bar{E}\}$. We consider $K_{p}(I-Q) N(\bar{E})$. Since $g:[0, \infty) \times \Re^{n} \longrightarrow \Re$ satisfies Caratheodory's conditions with respect to $L^{1}[0, \infty)$, there exist a Lebesgue integrable function $\varphi_{r}$ such that

$$
|N u(t)|=\mid g\left(t, u(t), u^{\prime}(t) \cdots u^{(n-1)}(t) \mid \leq \varphi_{r}(t) \text { a.e. } t \in(0, \infty)\right.
$$




$$
\begin{aligned}
&\|N u\|_{1} \leq \int_{0}^{\infty} \varphi_{r}(t) d t=\left\|\varphi_{r}\right\|_{1} \\
&\|Q N u\|_{1} \leq \int_{0}^{\infty}|Q N u(s)| d s=\int_{0}^{\infty} \mid h(t)\left[\int _ { 0 } ^ { \infty } \left(g\left(\tau, u(\tau), u^{\prime}(\tau) \ldots u^{(n-1)}(\tau)\right) d \tau\right.\right. \\
&-\int_{0}^{\xi} \int_{0}^{s}\left(g\left(\tau, u(\tau), u^{\prime}(\tau) \cdots u^{(n-1)}(\tau)\right) d \tau d A(s)\right] \mid d t \\
& \leq \int_{0}^{\infty}|h(t)|\left[\int_{0}^{\infty}\left|g\left(\tau, u(\tau), u^{\prime}(\tau) \ldots u^{(n-1)}(\tau)\right)\right| d \tau\right. \\
&\left.+\int_{0}^{\xi} \int_{0}^{s}\left|g\left(\tau, u(\tau), u^{\prime}(\tau) \ldots u^{(n-1)}(\tau)\right)\right| d \tau d A(s)\right] d t \\
& \leq \int_{0}^{\infty} \mid h(t)\left[\int_{0}^{\infty} \varphi_{r}(\tau) d \tau+\int_{0}^{\xi} \int_{0}^{s} \varphi(\tau) d \tau d A(s)\right] d t \\
& \leq\|h\|_{1}\left[\left\|\varphi_{r}\right\|_{1}+\left\|\varphi_{r}\right\|_{1} A(\xi)\right]=2\left\|\varphi_{r}\right\|_{1}\|h\|_{1}
\end{aligned}
$$

This shows that $Q N(\bar{E})$ is bounded. We now apply Lemma 2.1 to prove the compactness of $K_{p}(I-Q) N(\bar{E})$. Let $u \in \bar{E}$ then from the definition of $K_{p}(I-Q) N(u)$ together with (10), (11) and (12) we derive

$$
\begin{aligned}
\left\|K_{p}(I-Q) N u\right\| & \leq D_{n}\|(I-Q) N u\|_{1} \leq D_{n}\|N u\|_{1}+\|Q N u\|_{1} \\
& \leq D_{n}\left\|\varphi_{r}\right\|_{1}+2\left\|\varphi_{r}\right\|_{1}\|h\|_{1}
\end{aligned}
$$

$K_{p}(I-Q) N(\bar{E})$ is therefore uniformly bounded in $X$. Let $u \in E$ and $t_{1}, t_{2} \in[0, T], t_{1}<t_{2}$ with $T \in(0, \infty)$. We prove that $K_{p}(I-Q) N(\bar{E})$ is equicontinuous on every compact subset $[0, T]$ of $[0, \infty)$. We have

$$
\begin{aligned}
& \left|e^{-t_{2}}\left(K_{p}(I-Q) N u\right)^{(i)}\left(t_{2}\right)-e^{-t_{1}}\left(K_{p}(I-Q) N u\right)^{(i)}\left(t_{1}\right)\right|, 0 \leq i \leq n-2 \\
& =\left|\int_{t_{1}}^{t_{2}}\left[e^{-s}\left(K_{p}(I-Q) N u\right)^{(i)}(s)\right]^{\prime} d s\right| \\
& =\left|\int_{t_{1}}^{t_{2}}\left[-e^{-s}\left(K_{p}(I-Q) N u\right)^{(i)}(s)+e^{-s}\left(K_{p}(I-Q) N u\right)^{i+1}(s)\right] d s\right| \\
& \leq 2\left|\left(t_{1}-t_{2}\right)\right|\left\|K_{p}(I-Q) N u\right\| \leq 2\left|t_{1}-t_{2}\right|\left[D_{n}\left\|\varphi_{r}\right\|_{1}+2\left\|\varphi_{r}\right\|_{1}\|h\|_{1}\right] \\
& \longrightarrow 0 \text { as } t_{1} \rightarrow t_{2}
\end{aligned}
$$

For $i=n-1$, we obtain

$$
\begin{aligned}
& \left|e^{-t_{2}}\left(K_{p}(I-Q) N u\right)^{(n-1)}\left(t_{2}\right)-e^{-t_{1}}\left(K_{p}(I-Q) N u\right)^{(n-1)}\left(t_{1}\right)\right| \\
& =\left|e^{-t_{2}} \int_{0}^{t_{2}}(I-Q) N u(s) d s-e^{-t_{1}} \int_{0}^{t_{1}}(I-Q) N u(s) d s\right| \\
& \left.\leq \int_{0}^{t_{2}}\left(e^{-t_{1}}-e^{-t_{2}}\right)|(I-Q) N u(s)| d s+\int_{t_{1}}^{t_{2}} e^{-t_{1}}|(I-Q) N u(s)| d s\right]
\end{aligned}
$$




$$
\longrightarrow 0 \text { las } t_{1} \rightarrow t_{2}
$$

It follows that $K_{p}(I-Q) N(\bar{E})$ is equicontinuous on every compact subset of $[0, \infty)$. Next, we prove that $K_{p}(I-Q) N(\bar{E})$ is equicontinuous at infinity. For $u \in \bar{E}$ we have

$$
\begin{aligned}
\left|e^{-t}\left(K_{p}(I-Q) N u\right)(t)\right|= & \mid \frac{e^{-t}}{(n-1) !} \int_{0}^{t}(t-s)^{n-1}(I-Q) N u(s) d s \\
& -\frac{e^{-t}}{(n-1) !} \int_{0}^{\xi}(t-s)^{n-1}(I-Q) N u(s) d s \mid \\
& \leq e^{-t} t^{n-1} \int_{0}^{\infty}|(I-Q) N u(s)| d s+e^{-t} t^{n-1} \int_{0}^{\infty}|(I-Q) N u(s)| d s \\
& \leq 2 e^{-t} t^{n-1}\left(\|N u\|_{1}+\|Q N u\|_{1}\right) \leq 2 e^{-t} t^{n-1}\left[\left\|\varphi_{r}\right\|+2\left\|\varphi_{r}\right\|_{1}\|h\|_{1}\right] \\
& \longrightarrow 0 \text { as } t \rightarrow \infty .
\end{aligned}
$$

For $i=1,2, \ldots, n-1$ we have

$$
\begin{aligned}
\left|e^{-t}\left(K_{p}(I-Q) N u\right)^{(i)}(t)\right| & \leq \frac{e^{-t}}{(n-1-i) !} \int_{0}^{t}(t-s)^{n-1-i}|(I-Q) N u(s)| d s \\
& \leq e^{-t} t^{n-1-i} \int_{0}^{t}|(I-Q) N u(s)| d s \\
& \leq e^{-t} t^{n-1-i}\|(I-Q) N u\|_{1} \\
& \leq e^{-t} t^{n-1-i}\left[\left\|\varphi_{r}\right\|_{1}+2\left\|\varphi_{r}\right\|_{1}\|h\|_{1}\right] \\
& \longrightarrow 0 \text { as } t \rightarrow \infty .
\end{aligned}
$$

We conclude that $K_{p}(I-Q) N(\bar{E})$ is equiconvergence at $\infty$.

\section{Existence Results}

To establish the main existence results, we assume the following conditions

(R1) There exists functions $a_{i}(i=0,1, \ldots, n-1), b, r \in L^{1}[0, \infty)$ and constant $\theta \in[0,1)$ where $a_{i}, b, r:[0, \infty) \longrightarrow[0, \infty)$ are such that for all $\left(u_{0}, u_{1}, \ldots, u_{n-1}\right) \in \Re^{n}$, the following inequality is satisfied

$$
\mid g\left(t, u_{0}(t) \cdots u_{n-1}(t) \mid \leq e^{-t}\left(\sum_{i=0}^{n-1} a_{i}(t)\left|u_{i}(t)\right|+b(t)\left|u_{n-1}(t)\right|^{\theta}\right)+r(t)\right.
$$

(R2) There exists a constant $B_{1}>0$ such that for $u \in \operatorname{dom} L$ if $u^{(n-1)}(t)>B_{1}$ for all $t \in[0, \infty)$ we have $Q N u \neq 0$

(R3) There exists a constant $B_{2}>0$ such that for $u(t)=d t^{n-1} \in \operatorname{ker} L, d \in \Re$ with $|d|>\frac{B_{2}}{(n-1) !}$ then either 


$$
\begin{array}{r}
d \cdot h(t)\left[\int_{0}^{\infty} g\left(v, d v^{n-1}, d(n-1) v^{n-2} \cdots(n-1) ! d\right) d v\right. \\
-\int_{0}^{\xi} \int_{0}^{s} g\left(\tau, d \tau^{n-1}, d(n-1) \tau^{n-2} \cdots(n-1) ! d\right) d \tau d A(s)>0
\end{array}
$$

or

$$
\begin{array}{r}
d \cdot h(t)\left[\int_{0}^{\infty} g\left(v, d v^{n-1}, d(n-1) v^{n-2} \cdots(n-1) ! d\right) d v\right. \\
-\int_{0}^{\xi} \int_{0}^{s} g\left(\tau, d \tau^{n-1}, d(n-1) \tau^{n-2} \cdots(n-1) ! d\right) d \tau d A(s)<0
\end{array}
$$

Theorem 3.1: If $(R 1)-(R 3)$ hold, then the boundary value problem (1)-(2) has at least one solution in $C^{n-1}[0, \infty)$ provided

$$
\sum_{i=0}^{n-1}\left\|a_{i}\right\|_{1}<\frac{1}{2 D_{n}}
$$

Proof: Our goal is to construct an open bounded set $\Omega \subset X$ that satisfies assumption (1)-(3) of theorem 2.2 .

Let $\Omega_{1}=\{u \in \operatorname{dom} L \backslash \operatorname{ker} L, L u=\lambda N u$ for $\lambda \in(0,1]\}$.

For $u \in \Omega_{1}, u \notin \operatorname{ker} L$ and therefore $N u \in \operatorname{Im} L=\operatorname{ker} Q$. Thus $Q N u=0$ and by (R2) there exist $t_{0} \in[0, \infty)$ such that $\left|u^{(n-1)}\left(t_{0}\right)\right| \leq B_{1}$. We have

$$
\begin{aligned}
\left|u^{(n-1)}(0)\right| & =\left|u^{(n-1)}\left(t_{0}\right)-\int_{0}^{t_{0}} u^{(n)}(s) d s\right| \leq B_{1}+\int_{0}^{\infty}|N u(s)| d s \\
& =B_{1}+\|N u\|_{1}
\end{aligned}
$$

For $u \in \Omega_{1}, u \in d o m L \backslash \operatorname{ker} L$ and hence $(I-P) u \in d o m L \cap$ ker $P$ with $L P u=0$. Thus from (10) we obtain

$$
\|(I-P) u\|=\left\|K_{p} L(I-P) u\right\| \leq D_{n}\|L(I-P) u\|_{1} \leq D_{n}\|L u\|_{1} \leq D_{n}\|N u\|_{1}
$$

Using (18) and (19) we get

$$
\begin{aligned}
\|u\|=\|P u+(I-P) u\| \leq\|P u\|+\|\|(I-P u) \| & \leq D_{n}\left|u^{(n-1)}(0)\right|+D_{n}\|N u\|_{1} \\
& =D_{n}\left(B_{1}+\|N u\|_{1}\right)+D_{n}\|N u\|_{1} \\
& =D_{n} B_{1}+2 D_{n}\|N u\|_{1}
\end{aligned}
$$


Using (14) we have

$$
\begin{gathered}
\|N u\|_{1}=\int_{0}^{\infty} \mid g\left(s, u(s) \cdots u^{n-1}(s) \mid d s\right. \\
\leq \sum_{i=0}^{n-1} \int_{0}^{\infty}\left|a_{i}\right| e^{-s}\left|u^{(i)}(s)\right| d s+\int_{0}^{\infty}|b(s)| e^{-s}\left|u^{n-1}(s)\right|^{\theta} d s+\int_{0}^{\infty}|r(s)| d s \\
\leq \sum_{i=0}^{n-1}\left\|a_{i}\right\|_{1},\|u\|+\|b\|_{1},\|u\|^{\theta}+\|r\|_{1}
\end{gathered}
$$

From (20) we derive

$$
\begin{aligned}
\|u\| & \leq D_{n} B_{1}+2 D_{n}\left[\sum_{i=0}^{n-1}\left\|a_{i}\right\|_{1}\left\|u^{(i)}\right\|_{\infty} \mid\|b\|_{1}\left\|u^{(n-1)}\right\|_{\infty}^{\theta}+\|r\|_{1}\right] \\
& \leq D_{n} B_{1}+2 D_{n}\left[\sum_{i=0}^{n-1}\left\|a_{i}\right\|_{1}\|u\|+\|b\|_{1}\|u\|^{\theta}+\|r\|_{1}\right]
\end{aligned}
$$

i.e. $\left(1-2 D_{n} \sum_{i=0}^{n-1}\left\|a_{i}\right\|_{1}\right)\|u\| \leq 2 D_{n}\|b\|_{1}\|u\|^{\theta}+D_{n} B_{1}+2 D_{n}\|r\|_{1}$

Since $\theta \in[0,1)$ and condition (17), we conclude that there exists constant $M>0$ such that $\|u\| \leq M$. Therefore, $\Omega_{1}$ bounded.

Let $\Omega_{2}=\{u \in \operatorname{ker} L: N u \in \operatorname{Im} L\}$.

For $u \in \Omega_{2}, u \in \operatorname{ker} L=\left\{u \in \operatorname{dom} L: u=d t^{n-1}, d \in \Re, t \in[0, \infty)\right\}$ and $Q N u=0$. Therefore from (R2) there exist $t_{0} \in[0, \infty)$ such that $\left|u^{(n-1)}\left(t_{0}\right)\right|<B_{1}$ i.e, $(n-1) ! d \leq B_{1}$ which implies that $|d| \leq \frac{B_{1}}{(n-1) !}$.

Now for $u \in \Omega_{2}$

$$
\|u\|=|d| \max \left(\sup _{t \in[0, \infty)} e^{-t}\left|\left(t^{n-1}\right)^{(i)}\right|\right) \leq B_{1} D_{n}<\infty
$$

Therefore $\Omega_{2}$ is bounded in $X$.

If (15) holds, we set

$$
\Omega_{3}=\{u \in \operatorname{ker} L: \lambda J u+(1-\lambda) Q N u=0\}
$$

where $J$ is the isomorphism, $J: \operatorname{ker} L \rightarrow \operatorname{Im} Q$ defined by $J\left(d t^{n-1}\right)=d e^{-t} ; d \in \Re$.

For $u \in \Omega_{3}, u=d t^{n-1}$ and from (23) we get

$-\lambda J u=(1-\lambda) Q N u$

$-\lambda d e^{-t}=(1-\lambda) h(t)\left[\int_{0}^{\infty} N u(v) d v-\int_{0}^{\xi} \int_{0}^{s} N u(\tau) d \tau d A(s)\right]$ 
If $\lambda=1$, then $d=0$. However if $|d|>\frac{B_{1}}{(n-1) !}$ and $0<\lambda<1$

then from (15) we obtain

$$
-\lambda d^{2} e^{-t}=(1-\lambda) h(t) d \cdot\left[\int_{0}^{\infty} N u(v) d v-\int_{0}^{\xi} \int_{0}^{s} N u(\tau) d \tau d A(s)\right]>0
$$

which is a contradiction.

Similarly if $\Omega_{3}=\{u \in \operatorname{ker} L:-\lambda J u+(1-\lambda) Q N u=0\}$ we arrive at a similar contradiction using (16). Therefore, $\Omega_{3}$ is bounded.

Let $\Omega$ be open and bounded such that $\cup_{i=1}^{3} \Omega_{i} \subset \Omega$. It is easily seen that assumptions (1) and (2) of theorem 2.2 are satisfied. We now verify the third assumption. To do this, we apply the invariance under a homotopy of the degree. We define

$$
H(u, \lambda)= \pm \lambda J u+(1-\lambda) Q N u
$$

Since $\cup_{i=1}^{3} \Omega_{i} \subset \Omega$, we have that $H(u, \lambda) \neq 0$ for $u \in \operatorname{ker} L \cap \partial \Omega$. Hence

$$
\begin{aligned}
\operatorname{deg}\left(\left.Q N\right|_{\operatorname{ker} L \cap \partial \Omega}, \Omega \cap \operatorname{ker} L, 0\right) & =\operatorname{deg}(H(0,1), \Omega \cap \operatorname{ker} L, 0) \\
& =\operatorname{deg}( \pm J, \Omega \cap \operatorname{ker} L, 0) \neq 0
\end{aligned}
$$

Therefore by theorem 2.1 $L u=N u$ has at least one solution in $\operatorname{dom} L \cap \bar{\Omega}$ i.e. (1) - (2) has at least one solution in $X$

\section{Conclusion}

This paper has established conditions for the existence of solutions for the resonant boundary value problems (1) - (2); using coincidence degree theory. the results obtained here are new and complements existing results for higher order boundary value problems on infinite intervals.

\section{Acknowledgements}

Authors are grateful to Covenant University for financial assistance and the reviewers for their useful comments.

\section{References}

[1] R.P. Agarwal. Boundary value problem for Higher order differential equations, World Scientific, Singapore 1986.

[2] R.P. Agarwal, D.O. O'Regan. Infinity interval problems for difference and integral equations, Kluwer Academic Publisher. Derdrecht 2001.

[3] A.V. Bicadze and A.A. Samarskii. Some elementary generalisations of linear elliptic boundary value problems, Dokhady Nauk SSSR, 185(1969) 739-749. 
[4] Y. Cui. Solvability of second order boundary value problems at resonance involving integral conditions, Electron J. Differential equation, 45(2012) 1-9.

[5] Z. I. Du, X.I. Lin, H.G. Ge. Some higher-order multipoint boundary value problems at resonance, J. Comput. Appl. Math. 177 (2015), 55-65.

[6] D. Franco, G. Infante, M. Zima. Second order nonlocal boundary value problems at resonance Math. Nachr. 284 (7) (2011).

[7] A. Frioui, A Guezane-Lakoud, R. Khaldi. Higher order boundary value problems at resonance on an unbounded interval Electronic, J. Diff. Equation, 29(2016), 1-10.

[8] S.A. Iyase On a third-order boundary value problem at resonance on the half-line, Arabian Journal of Mathematics, 8(2019), 43-53. https://doi.org/10.1007/s40065018-0209-5.

[9] S.A. Iyase and O.F. Imaga. On a singular second-order multipoint boundary value problem at Resonance, International Journal of Differential Equations, (2017), 1-6. ID8579065.

[10] G.L. Karakistas and P.Isamatos. Sufficient conditions for the existence of nonnegative solutions of a nonlcal boundary value problem, Applied Mathematics Letters, 15(4)(2002), 401-407.

[11] A.M. Krosnosel'skii, J. Mawhin. On some higher order boundary value problems at resonance, Nonlinear Anal. 24 (1995), 1411-1148.

[12] H.R. Lian, H.H. Pang, W.G. Ge. Solvability for second order three point boundaryvalue problems at resonance on a half-line. J. Math. Anal. Appl. 337 (2008), 1171-1181.

[13] X.J. Lin, Z.J. Du, W.G. Ge. Solvability of multipoint boundary value problems at resonance for higher order ordinary differential equations, Comput. Math. Appl. 49 (2005), 1-11.

[14] X. Lin. Existence of solutions to a nonlocal boundary value problem with nonlinear growth. Boundary value problems.(2011)

doi : $10.1155 / 2011 / 416416$.

[15] Y. Liu. W.Ge Solutions of a multipoint boundary value problem for higher order differential equations at resonance. Tamakang. Journal of Maths 36(2)(2005), 119-130.

[16] Y. Liu, D.Li, M. Fang. Solvability for second order m-point boundary value problems on the half-line. Electron J. of diff. equation, 13(2009), 1-11.

[17] J. Mawhin. Topological degree methods in nonlinear boundary value problems. NSFCBMS. Regional Conference Series in Math. Vol. 40. Americ. Math. Soc. Providence RI 1979. 
[18] J.R.L. Webb, G. Infante. Positive solutions of nonlocal boundary value problems involving integral conditions. Nonlinear differential equations. Appl. 15(2008), 45-67.

[19] M. Zima. On positive solutions of boundary value problems on the half-line. J. Math. Anal. Appl. 259 (2001), 127-136 